DESY 05-249

ISSN 0418-9833

December 2005

\title{
First Measurement of Charged Current Cross Sections at HERA with Longitudinally Polarised Positrons
}

\author{
H1 Collaboration
}

\begin{abstract}
Data taken with positrons of different longitudinal polarisation states in collision with unpolarised protons at HERA are used to measure the total cross sections of the charged current process, $e^{+} p \rightarrow \bar{\nu} X$, for negative four-momentum transfer squared $Q^{2}>400 \mathrm{GeV}^{2}$ and inelasticity $y<0.9$. Together with the corresponding cross section obtained from the previously published unpolarised data, the polarisation dependence of the charged current cross section is measured for the first time at high $Q^{2}$ and found to be in agreement with the Standard Model prediction.
\end{abstract}

Dedicated to the memory of our dear friend and colleague, Alexei Babaev

Submitted to Phys. Lett. B 


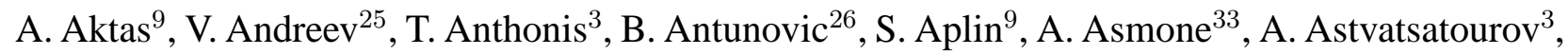
A. Babaev ${ }^{24,}$, S. Backovic ${ }^{30}$, J. Bähr ${ }^{38}$, A. Baghdasaryan ${ }^{37}$, P. Baranov $^{25}$, E. Barrelet ${ }^{29}$, W. Bartel ${ }^{9}$, S. Baudrand ${ }^{27}$, S. Baumgartner ${ }^{39}$, J. Becker ${ }^{40}$, M. Beckingham ${ }^{9}$, O. Behnke ${ }^{12}$, O. Behrendt ${ }^{6}$, A. Belousov ${ }^{25}$, Ch. Berger ${ }^{1}$, N. Berger ${ }^{39}$, J.C. Bizot ${ }^{27}$, M.-O. Boenig ${ }^{6}$, V. Boudry ${ }^{28}$, J. Bracinik ${ }^{26}$, G. Brandt ${ }^{12}$, V. Brisson ${ }^{27}$, D. Bruncko ${ }^{15}$, F.W. Büsser ${ }^{10}$, A. Bunyatyan ${ }^{11,37}$, G. Buschhorn ${ }^{26}$, L. Bystritskaya ${ }^{24}$, A.J. Campbell ${ }^{9}$, F. Cassol-Brunner ${ }^{21}$, K. Cerny ${ }^{32}$, V. Cerny ${ }^{15,46}$, V. Chekelian ${ }^{26}$, J.G. Contreras ${ }^{22}$, J.A. Coughlan ${ }^{4}$, B.E. Cox ${ }^{20}$, G. Cozzika ${ }^{8}$, J. Cvach ${ }^{31}$, J.B. Dainton ${ }^{17}$, W.D. Dau ${ }^{14}$, K. Daum ${ }^{36,42}$, Y. de Boer ${ }^{24}$, B. Delcourt ${ }^{27}$, M. Del Degan ${ }^{39}$, A. De Roeck ${ }^{9,44}$, K. Desch ${ }^{10}$, E.A. De Wolf ${ }^{3}$, C. Diaconu ${ }^{21}$, V. Dodonov ${ }^{11}$, A. Dubak ${ }^{30,45}$, G. Eckerlin ${ }^{9}$, V. Efremenko ${ }^{24}$, S. Egli ${ }^{35}$, R. Eichler ${ }^{35}$, F. Eisele ${ }^{12}$, E. Elsen ${ }^{9}$, W. Erdmann ${ }^{39}$, S. Essenov ${ }^{24}$, A. Falkewicz ${ }^{5}$, P.J.W. Faulkner ${ }^{2}$, L. Favart ${ }^{3}$, A. Fedotov ${ }^{24}$, J. Feltesse ${ }^{8}$, J. Ferencei ${ }^{15}$, L. Finke ${ }^{10}$, M. Fleischer ${ }^{9}$, P. Fleischmann ${ }^{9}$, G. Flucke ${ }^{33}$, A. Fomenko ${ }^{25}$, I. Foresti ${ }^{40}$, G. Franke ${ }^{9}$, T. Frisson ${ }^{28}$, E. Gabathuler ${ }^{17}$, E. Garutti ${ }^{9}$, J. Gayler ${ }^{9}$, C. Gerlich ${ }^{12}$, S. Ghazaryan ${ }^{37}$, S. Ginzburgskaya ${ }^{24}$, A. Glazov ${ }^{9}$, I. Glushkov ${ }^{38}$, L. Goerlich ${ }^{5}$, M. Goettlich ${ }^{9}$, N. Gogitidze ${ }^{25}$, S. Gorbounov ${ }^{38}$, C. Goyon $^{21}$, C. Grab $^{39}$, T. Greenshaw ${ }^{17}$, M. Gregori ${ }^{18}$, B.R. Grell ${ }^{9}$, G. Grindhammer ${ }^{26}$, C. Gwilliam ${ }^{20}$, D. Haidt ${ }^{9}$, L. Hajduk ${ }^{5}$, M. Hansson ${ }^{19}$, G. Heinzelmann ${ }^{10}$, R.C.W. Henderson ${ }^{16}$, H. Henschel ${ }^{38}$, G. Herrera ${ }^{23}$, M. Hildebrandt ${ }^{35}$, K.H. Hiller ${ }^{38}$, D. Hoffmann ${ }^{21}$, R. Horisberger ${ }^{35}$, A. Hovhannisyan ${ }^{37}$, T. Hreus ${ }^{3,43}$, S. Hussain ${ }^{18}$, M. Ibbotson ${ }^{20}$, M. Ismail ${ }^{20}$, M. Jacquet ${ }^{27}$, L. Janauschek ${ }^{26}$, X. Janssen ${ }^{9}$, V. Jemanov ${ }^{10}$, L. Jönsson ${ }^{19}$, D.P. Johnson ${ }^{3}$, A.W. Jung ${ }^{13}$, H. Jung ${ }^{19,9}$, M. Kapichine ${ }^{7}$, J. Katzy ${ }^{9}$, I.R. Kenyon ${ }^{2}$, C. Kiesling ${ }^{26}$, M. Klein ${ }^{38}$, C. Kleinwort ${ }^{9}$, T. Klimkovich ${ }^{9}$, T. Kluge ${ }^{9}$, G. Knies ${ }^{9}$, A. Knutsson ${ }^{19}$, V. Korbel ${ }^{9}$, P. Kostka ${ }^{38}$, K. Krastev ${ }^{9}$, J. Kretzschmar ${ }^{38}$, A. Kropivnitskaya ${ }^{24}$, K. Krüger ${ }^{13}$, J. Kückens ${ }^{9}$, M.P.J. Landon ${ }^{18}$, W. Lange ${ }^{38}$, T. Laštovička ${ }^{38,32}$, G. Laštovička-Medin ${ }^{30}$, P. Laycock ${ }^{17}$, A. Lebedev ${ }^{25}$, G. Leibenguth ${ }^{39}$,

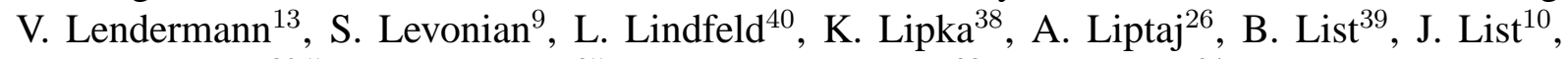
E. Lobodzinska ${ }^{38,5}$, N. Loktionova ${ }^{25}$, R. Lopez-Fernandez ${ }^{23}$, V. Lubimov ${ }^{24}$, A.-I. Lucaci-Timoce ${ }^{9}$, H. Lueders ${ }^{10}$, D. Lüke ${ }^{6,9}$, T. Lux ${ }^{10}$, L. Lytkin ${ }^{11}$, A. Makankine ${ }^{7}$, N. Malden ${ }^{20}$, E. Malinovski ${ }^{25}$, S. Mangano $^{39}$, P. Marage ${ }^{3}$, R. Marshall ${ }^{20}$, M. Martisikova ${ }^{9}$, H.-U. Martyn ${ }^{1}$, S.J. Maxfield ${ }^{17}$, D. Meer ${ }^{39}$, A. Mehta ${ }^{17}$, K. Meier ${ }^{13}$, A.B. Meyer ${ }^{9}$, H. Meyer $^{36}$, J. Meyer $^{9}$, V. Michels ${ }^{9}$, S. Mikocki $^{5}$, I. Milcewicz-Mika ${ }^{5}$, D. Milstead ${ }^{17}$, D. Mladenov ${ }^{34}$, A. Mohamed ${ }^{17}$, F. Moreau ${ }^{28}$, A. Morozov ${ }^{7}$, J.V. Morris ${ }^{4}$, M.U. Mozer ${ }^{12}$, K. Müller ${ }^{40}$, P. Murín ${ }^{15,43}$, K. Nankov ${ }^{34}$, B. Naroska ${ }^{10}$, Th. Naumann ${ }^{38}$, P.R. Newman ${ }^{2}$, C. Niebuhr ${ }^{9}$, A. Nikiforov ${ }^{26}$, G. Nowak ${ }^{5}$, M. Nozicka ${ }^{32}$, R. Oganezov $^{37}$, B. Olivier ${ }^{26}$, J.E. Olsson ${ }^{9}$, S. Osman ${ }^{19}$, D. Ozerov ${ }^{24}$, V. Palichik ${ }^{7}$, I. Panagoulias ${ }^{9}$, T. Papadopoulou ${ }^{9}$, C. Pascaud ${ }^{27}$, G.D. Patel ${ }^{17}$, H. Peng ${ }^{9}$, E. Perez ${ }^{8}$, D. Perez-Astudillo ${ }^{22}$, A. Perieanu ${ }^{9}$, A. Petrukhin ${ }^{24}$, D. Pitzl ${ }^{9}$, R. Plačakytė ${ }^{26}$, B. Portheault ${ }^{27}$, B. Povh ${ }^{11}$, P. Prideaux ${ }^{17}$, A.J. Rahmat ${ }^{17}$, N. Raicevic ${ }^{30}$, B. Reisert ${ }^{47}$, P. Reimer ${ }^{31}$, A. Rimmer ${ }^{17}$, C. Risler ${ }^{9}$, E. Rizvi ${ }^{18}$, P. Robmann ${ }^{40}$, B. Roland ${ }^{3}$, R. Roosen ${ }^{3}$, A. Rostovtsev $^{24}$, Z. Rurikova ${ }^{26}$, S. Rusakov ${ }^{25}$, F. Salvaire ${ }^{10}$, D.P.C. Sankey ${ }^{4}$, E. Sauvan ${ }^{21}$, S. Schätzel ${ }^{9}$, S. Schmidt ${ }^{9}$, S. Schmitt ${ }^{9}$, C. Schmitz ${ }^{40}$, L. Schoeffel ${ }^{8}$, A. Schöning ${ }^{39}$, H.-C. SchultzCoulon $^{13}$, K. Sedlák ${ }^{31}$, F. Sefkow ${ }^{9}$, R.N. Shaw-West ${ }^{2}$, I. Sheviakov ${ }^{25}$, L.N. Shtarkov ${ }^{25}$, T. Sloan ${ }^{16}$, P. Smirnov ${ }^{25}$, Y. Soloviev ${ }^{25}$, D. South ${ }^{9}$, V. Spaskov ${ }^{7}$, A. Specka ${ }^{28}$, M. Steder ${ }^{10}$, B. Stella ${ }^{33}$, J. Stiewe ${ }^{13}$, I. Strauch ${ }^{9}$, U. Straumann ${ }^{40}$, D. Sunar ${ }^{3}$, V. Tchoulakov ${ }^{7}$, G. Thompson ${ }^{18}$, P.D. Thompson ${ }^{2}$, F. Tomasz ${ }^{15}$, D. Traynor ${ }^{18}$, P. Truöl ${ }^{40}$, I. Tsakov ${ }^{34}$, G. Tsipolitis ${ }^{9,41}$, I. Tsurin ${ }^{9}$, J. Turnau ${ }^{5}$, E. Tzamariudaki ${ }^{26}$, K. Urban ${ }^{13}$, M. Urban ${ }^{40}$, A. Usik ${ }^{25}$, D. Utkin ${ }^{24}$, A. Valkárová ${ }^{32}$, C. Vallée ${ }^{21}$, P. Van Mechelen ${ }^{3}$, A. Vargas Trevino ${ }^{6}$, Y. Vazdik ${ }^{25}$, C. Veelken ${ }^{17}$, S. Vinokurova ${ }^{9}$, V. Volchinski ${ }^{37}$, K. Wacker ${ }^{6}$, J. Wagner ${ }^{9}$, G. Weber ${ }^{10}$, R. Weber ${ }^{39}$, D. Wegener ${ }^{6}$, C. Werner ${ }^{12}$, M. Wessels ${ }^{9}$, B. Wessling ${ }^{9}$, C. Wigmore ${ }^{2}$, Ch. Wissing ${ }^{6}$, R. Wolf ${ }^{12}$, E. Wünsch ${ }^{9}$, S. Xella ${ }^{40}$, W. Yan ${ }^{9}$, V. Yeganov ${ }^{37}$, J. Žáček ${ }^{32}$, J. Zálešák ${ }^{31}$, Z. Zhang ${ }^{27}$, A. Zhelezov ${ }^{24}$, A. Zhokin ${ }^{24}$, Y.C. Zhu ${ }^{9}$, J. Zimmermann ${ }^{26}$, T. Zimmermann ${ }^{39}$, H. Zohrabyan ${ }^{37}$, and F. Zomer ${ }^{27}$ 
${ }^{1}$ I. Physikalisches Institut der RWTH, Aachen, Germany ${ }^{a}$

${ }^{2}$ School of Physics and Astronomy, University of Birmingham, Birmingham, $U K^{b}$

${ }^{3}$ Inter-University Institute for High Energies ULB-VUB, Brussels; Universiteit Antwerpen, Antwerpen; Belgium ${ }^{c}$

${ }^{4}$ Rutherford Appleton Laboratory, Chilton, Didcot, $U K^{b}$

${ }^{5}$ Institute for Nuclear Physics, Cracow, Poland ${ }^{d}$

${ }^{6}$ Institut für Physik, Universität Dortmund, Dortmund, Germany ${ }^{a}$

${ }^{7}$ Joint Institute for Nuclear Research, Dubna, Russia

${ }^{8}$ CEA, DSM/DAPNIA, CE-Saclay, Gif-sur-Yvette, France

${ }^{9}$ DESY, Hamburg, Germany

${ }^{10}$ Institut für Experimentalphysik, Universität Hamburg, Hamburg, Germany ${ }^{a}$

${ }^{11}$ Max-Planck-Institut für Kernphysik, Heidelberg, Germany

${ }^{12}$ Physikalisches Institut, Universität Heidelberg, Heidelberg, Germany ${ }^{a}$

${ }^{13}$ Kirchhoff-Institut für Physik, Universität Heidelberg, Heidelberg, Germany ${ }^{a}$

${ }^{14}$ Institut für Experimentelle und Angewandte Physik, Universität Kiel, Kiel, Germany

${ }^{15}$ Institute of Experimental Physics, Slovak Academy of Sciences, Košice, Slovak Republic ${ }^{f}$

${ }^{16}$ Department of Physics, University of Lancaster, Lancaster, $U K^{b}$

${ }^{17}$ Department of Physics, University of Liverpool, Liverpool, $U K^{b}$

${ }^{18}$ Queen Mary and Westfield College, London, $U K^{b}$

${ }^{19}$ Physics Department, University of Lund, Lund, Sweden ${ }^{g}$

${ }^{20}$ Physics Department, University of Manchester, Manchester, $U K^{b}$

${ }^{21}$ CPPM, CNRS/IN2P3 - Univ. Mediterranee, Marseille - France

${ }^{22}$ Departamento de Fisica Aplicada, CINVESTAV, Mérida, Yucatán, México ${ }^{k}$

${ }^{23}$ Departamento de Fisica, CINVESTAV, México ${ }^{k}$

${ }^{24}$ Institute for Theoretical and Experimental Physics, Moscow, Russial

${ }^{25}$ Lebedev Physical Institute, Moscow, Russia ${ }^{e}$

${ }^{26}$ Max-Planck-Institut für Physik, München, Germany

${ }^{27}$ LAL, Université de Paris-Sud, IN2P3-CNRS, Orsay, France

${ }^{28}$ LLR, Ecole Polytechnique, IN2P3-CNRS, Palaiseau, France

${ }^{29}$ LPNHE, Universités Paris VI and VII, IN2P3-CNRS, Paris, France

${ }^{30}$ Faculty of Science, University of Montenegro, Podgorica, Serbia and Montenegro ${ }^{e}$

${ }^{31}$ Institute of Physics, Academy of Sciences of the Czech Republic, Praha, Czech Republic ${ }^{i}$

${ }^{32}$ Faculty of Mathematics and Physics, Charles University, Praha, Czech Republic ${ }^{i}$

${ }^{33}$ Dipartimento di Fisica Università di Roma Tre and INFN Roma 3, Roma, Italy

${ }^{34}$ Institute for Nuclear Research and Nuclear Energy, Sofia, Bulgaria ${ }^{e}$

${ }^{35}$ Paul Scherrer Institut, Villigen, Switzerland

${ }^{36}$ Fachbereich C, Universität Wuppertal, Wuppertal, Germany

${ }^{37}$ Yerevan Physics Institute, Yerevan, Armenia

${ }^{38}$ DESY, Zeuthen, Germany

${ }^{39}$ Institut für Teilchenphysik, ETH, Zürich, Switzerland ${ }^{j}$

${ }^{40}$ Physik-Institut der Universität Zürich, Zürich, Switzerland ${ }^{j}$

${ }^{41}$ Also at Physics Department, National Technical University, Zografou Campus, GR-15773 Athens, Greece

${ }^{42}$ Also at Rechenzentrum, Universität Wuppertal, Wuppertal, Germany 
${ }^{43}$ Also at University of P.J. Šafárik, Košice, Slovak Republic

${ }^{44}$ Also at CERN, Geneva, Switzerland

${ }^{45}$ Also at Max-Planck-Institut für Physik, München, Germany

${ }^{46}$ Also at Comenius University, Bratislava, Slovak Republic

${ }^{47}$ Now at Fermi National Accelerator Laboratory, Batavia, USA

$\dagger$ Deceased

${ }^{a}$ Supported by the Bundesministerium für Bildung und Forschung, FRG, under contract numbers 05 H1 1GUA /1, 05 H1 1PAA /1, 05 H1 1PAB /9, 05 H1 1PEA /6, 05 H1 1VHA /7 and 05 HI 1 VHB $/ 5$

${ }^{b}$ Supported by the UK Particle Physics and Astronomy Research Council, and formerly by the UK Science and Engineering Research Council

${ }^{c}$ Supported by FNRS-FWO-Vlaanderen, IISN-IIKW and IWT and by Interuniversity Attraction Poles Programme, Belgian Science Policy

${ }^{d}$ Partially Supported by the Polish State Committee for Scientific Research, SPUB/DESY/P003/DZ 118/2003/2005

e Supported by the Deutsche Forschungsgemeinschaft

${ }^{f}$ Supported by VEGA SR grant no. 2/4067/24

${ }^{g}$ Supported by the Swedish Natural Science Research Council

${ }^{i}$ Supported by the Ministry of Education of the Czech Republic under the projects LC527 and INGO-1P05LA259

${ }^{j}$ Supported by the Swiss National Science Foundation

${ }^{k}$ Supported by CONACYT, México, grant 400073-F

${ }^{l}$ Partially Supported by Russian Foundation for Basic Research, grants 03-02-17291 and 0402-16445 


\section{Introduction}

In autumn 2003 the HERA accelerator started operation of the second phase of its $e p$ collider programme. The $e^{+} p$ data collected by the H1 and ZEUS experiments since then were taken with a longitudinally polarised positron beam for the first time. Measurements of deep inelastic scattering (DIS) with polarised leptons on protons allow the parton distribution functions (PDFs) of the proton to be further constrained through polarisation asymmetries [1] and specific tests of the electroweak (EW) parts of the Standard Model to be performed [2, 3]. In particular, the measurements presented here extend the tests of the $\mathrm{V}-\mathrm{A}$ structure of charged current interactions from low $Q^{2}$ [4] into the high $Q^{2}$ regime, where $Q^{2}$ is the negative four-momentum transfer squared.

At HERA DIS proceeding via charged currents (CC), ep $\rightarrow \nu X$, and neutral currents (NC), $e p \rightarrow e X$, can be measured accurately $[5,6]$. The polarisation dependence of the CC and NC cross sections is fixed within the Standard Model framework. Specifically, the Standard Model predicts, from the absence of right handed charged currents, that the $\mathrm{CC} e^{+} p$ cross section is directly proportional to the fraction of right handed positrons in the beam.

In this paper first measurements of the charged current total cross sections, $\sigma_{\mathrm{CC}}^{\text {tot }}$, are reported for two values of longitudinal polarisation, $P_{e}=\left(N_{R}-N_{L}\right) /\left(N_{R}+N_{L}\right)$, with $N_{R}\left(N_{L}\right)$ being the number of right (left) handed positrons in the beam. The corresponding data sets are termed the $R$ and $L$ data sets respectively. The $R$ data set has a luminosity weighted mean polarisation value of $(33.6 \pm 0.7) \%$ and an integrated luminosity value of $26.9 \pm 0.6 \mathrm{pb}^{-1}$. The corresponding numbers for the $L$ data set are $(-40.2 \pm 1.1) \%$ and $20.7 \pm 0.5 \mathrm{pb}^{-1}$. In both data sets the incident positron beam energy is $27.5 \mathrm{GeV}$, whilst the unpolarised proton beam energy is $920 \mathrm{GeV}$. This yields a centre-of-mass energy of $\sqrt{s}=318 \mathrm{GeV}$.

The measurements presented here, as well as the corresponding one obtained using the published unpolarised data, are compared to Standard Model expectations and a linear fit to $\sigma_{\mathrm{CC}}^{\text {tot }}$ as a function of $P_{e}$ is performed. The result of the fit is used to derive a cross section for a fully left handed positron beam corresponding to $P_{e}=-1$.

\section{Charged Current Cross Section}

The measured double differential CC cross section for collisions of polarised positrons with unpolarised protons, corrected for QED radiative effects, may be expressed as

$$
\frac{\mathrm{d}^{2} \sigma_{\mathrm{CC}}}{\mathrm{d} x \mathrm{~d} Q^{2}}=\left(1+P_{e}\right) \frac{G_{F}^{2}}{4 \pi x}\left[\frac{M_{W}^{2}}{M_{W}^{2}+Q^{2}}\right]^{2}\left(Y_{+} W_{2}-Y_{-} x W_{3}-y^{2} W_{L}\right) \cdot\left(1+\delta_{\text {weak }}^{\mathrm{CC}}\right),
$$

where $x$ is the Bjorken $x$ variable and $y$ characterises the inelasticity of the interaction. The Fermi constant $G_{F}$ is defined [7] using the weak boson masses. Other quantities in Eq.(1) include $M_{W}$, the mass of the $W$ boson, $W_{2}, x W_{3}$ and $W_{L}, C C$ structure functions, and $\delta_{\text {weak }}^{\text {CC }}$, the weak radiative corrections. The helicity dependences of the weak interaction are contained in $Y_{ \pm}=1 \pm(1-y)^{2}$. In the quark parton model (QPM), where $W_{L} \equiv 0$, the structure functions 
$W_{2}$ and $x W_{3}$ for $e^{+} p$ scattering may be expressed as the sum and difference of the quark and anti-quark momentum distributions, $x q\left(x, Q^{2}\right)$ and $x \bar{q}\left(x, Q^{2}\right)$ :

$$
\begin{aligned}
W_{2} & =x(\bar{u}+\bar{c}+d+s), \\
x W_{3} & =x(-\bar{u}-\bar{c}+d+s) .
\end{aligned}
$$

The total cross section, $\sigma_{\mathrm{CC}}^{\text {tot }}$, is defined as the integrated cross section in the kinematic region $Q^{2}>400 \mathrm{GeV}^{2}$ and $y<0.9$. From Eq.(1) it can be seen that the cross section has a linear dependence on the polarisation of the positron beam $P_{e}$. For a fully left handed positron beam, $P_{e}=-1$, the cross section is identically zero in the Standard Model.

\section{Experimental Technique}

At HERA transverse polarisation of the positron beam arises naturally through synchrotron radiation via the Sokolov-Ternov effect [8]. In 2000 a pair of spin rotators was installed in the beamline on either side of the $\mathrm{H} 1$ detector, allowing transversely polarised positrons to be rotated into longitudinally polarised states and back again. The degree of polarisation is constant around the HERA ring and is continuously measured using two independent polarimeters LPOL [9] and TPOL [10]. The polarimeters are situated in beamline sections in which the beam leptons have longitudinal and transverse polarisations respectively. Both measurements rely on an asymmetry in the energy spectrum of left and right handed circularly polarised photons undergoing Compton scattering with the positron beam. The TPOL measurement uses in addition a spatial asymmetry. The LPOL polarimeter measurements are used when available and TPOL measurements otherwise.

The H1 detector components most relevant to this analysis are the liquid argon (LAr) calorimeter, which measures the positions and energies of charged and neutral particles over the polar ${ }^{1}$ angular range $4^{\circ}<\theta<154^{\circ}$, and the inner tracking detectors, which measure the angles and momenta of charged particles over the range $7^{\circ}<\theta<165^{\circ}$. A full description of the detector can be found in [11].

Simulated DIS events are used in order to determine acceptance corrections. DIS processes are generated using the DJANGO [12] Monte Carlo (MC) simulation program, which is based on LEPTO [13] for the hard interaction and HERACLES [14] for single photon emission and virtual EW corrections. LEPTO combines $\mathcal{O}\left(\alpha_{s}\right)$ matrix elements with higher order QCD effects using the colour dipole model as implemented in ARIADNE [15]. The JETSET program [16] is used to simulate the hadronisation process. In the event generation the DIS cross section is calculated using the H1 PDF 2000 [5] parametrisation for the proton PDFs.

The dominant $e p$ background contribution arises from photoproduction processes. These are simulated using the PYTHIA [17] MC with leading order PDFs for the proton taken from CTEQ [18] and for the photon from GRV [19]. Further backgrounds from NC DIS, QEDCompton scattering, lepton pair production, prompt photon production and heavy gauge boson $\left(W^{ \pm}, Z^{0}\right)$ production are also simulated; their final contribution to the analysis sample is small. Further details are given in [5].

\footnotetext{
${ }^{1}$ The polar angle $\theta$ is defi ned with respect to the positive $z$ axis, the direction of the incident proton beam.
} 
The detector response to events produced by the generation programs is simulated in detail using a program based on GEANT [20]. These simulated events are then subjected to the same reconstruction and analysis chain as the data.

The selection of CC interactions follows closely that of the previously published analysis of unpolarised data from H1 [5] and is briefly described below. The CC events are characterised as having large unbalanced transverse momentum, $P_{T, h}$, attributed to the undetected neutrino. The quantity $P_{T, h}$ is determined from $P_{T, h}=\sqrt{\left(\sum_{i} p_{x, i}\right)^{2}+\left(\sum_{i} p_{y, i}\right)^{2}}$, where the summation is performed over all particles of the hadronic final state. The hadronic final state particles are reconstructed using a combination of tracks and calorimeter deposits in an energy flow algorithm that avoids double counting [21].

The CC kinematic quantities are determined from the hadonic final state [22] using the relations

$$
y_{h}=\frac{E_{h}-p_{z, h}}{2 E_{e}}, \quad Q_{h}^{2}=\frac{P_{T, h}^{2}}{1-y_{h}}, \quad x_{h}=\frac{Q_{h}^{2}}{s y_{h}}
$$

where $E_{h}-p_{z, h} \equiv \sum_{i}\left(E_{i}-p_{z, i}\right)$ and $E_{e}$ is the incident positron beam energy.

NC interactions are also studied as they provide an accurate and high statistics data sample with which to check the detector response. The selection of NC interactions is based mainly on the requirement of an identified scattered positron in the LAr calorimeter, with an energy $E_{e}^{\prime}>11 \mathrm{GeV}$. The NC sample is used to carry out an in-situ calibration of the electromagnetic and hadronic energy scales of the LAr calorimeter using the method described in [5]. The hadronic calibration procedure is based on the balance of the transverse energy of the positrons with that of the hadronic final state. The calibration procedure gives good agreement between data and simulation within an estimated uncertainty of $2 \%$.

In addition, NC events are used for studies of systematic uncertainties in the charged current analysis. The data are processed such that all information from the scattered positron is suppressed, providing the so-called pseudo-CC sample [21, 23, 24]. This sample mimics CC interactions allowing trigger and selection efficiencies to be checked with high statistical precision and independently of the MC simulation.

\section{Measurement Procedure}

Candidate CC interactions are selected by requiring $P_{T, h}>12 \mathrm{GeV}$ and a reconstructed vertex within $35 \mathrm{~cm}$ in $z$ of the nominal interaction point. In order to ensure high efficiency of the trigger and good kinematic resolution the analysis is further restricted to the domain of $0.03<$ $y_{h}<0.85$. The $e p$ background is dominantly due to photoproduction events, in which the scattered positron escapes undetected in the backward direction and transverse momentum is missing due to fluctuations in the detector response or undetected particles. This background is suppressed exploiting the correlation between $P_{T, h}$ and the ratio $V_{a p} / V_{p}$ of transverse energy flow anti-parallel and parallel to the hadronic final state transverse momentum vector [21, 23, 24]. The suppression cuts are different for the $R$ and $L$ data sets as the relative photoproduction contributions differ in the two samples. The residual ep background is negligible for most of the measured kinematic domain. The simulation is used to estimate this contribution, which is 
subtracted statistically from the CC data sample. Non-ep background is rejected by searching for typical cosmic ray and beam-induced background event topologies [21, 23, 24]. The final $R$ $(L)$ CC data sample amounts to $\simeq 700(\simeq 200)$ events.

The $P_{T, h}$ and $E_{h}-p_{z, h}$ distributions of the selected events are shown in Fig. 1a,b for the $R$ sample and in Fig. 1c,d for the $L$ sample. The simulation provides a good description of the data. The contribution of background photoproduction processes is small and has the largest influence at low $P_{T, h}$.

Events with $Q_{h}^{2}>400 \mathrm{GeV}^{2}$ are used to measure the cross sections, which correspond to the kinematic region $Q^{2}>400 \mathrm{GeV}^{2}$ and $y<0.9$ and thus are corrected for the effects of the analysis cuts. The correction factor is calculated to be 1.067 using the H1 PDF 2000 parametrisation.

The systematic uncertainties on the cross section measurements are discussed briefly below (see [21, 23, 24] and references therein for more details). Positive and negative variations of one standard deviation of each error source are found to yield errors which are symmetric to a good approximation. The systematic uncertainties of each source are taken to be fully correlated between the cross section measurements unless stated otherwise.

- An uncertainty of $2 \%$ is assigned to the hadronic energy measured in the LAr calorimeter, of which $1 \%$ is considered as a correlated component to the uncertainty. This results in a total uncertainty of $1.3 \%$ on the cross section measurements.

- A $10 \%$ uncertainty is assigned to the amount of energy in the LAr calorimeter attributed to noise, which gives rise to a systematic error of $0.3 \%$ on the cross section measurements.

- The variation of cuts against photoproduction on $V_{a p} / V_{p}$ and $P_{T, h}$ has an effect on the cross sections of $0.6 \%$.

- A $30 \%$ uncertainty on the subtracted $e p$ background is determined from a comparison of data and simulation after relaxing the anti-photoproduction cuts, such that the sample is dominated by photoproduction events. This results in a systematic error of $0.5 \%(1 \%)$ on the cross section of the $R(L)$ data.

- The non-ep background finders introduce an inefficiency for CC events. The associated uncertainty is estimated using pseudo-CC data and found to depend on $y$. An uncertainty of $2 \%$ is applied for $y<0.1$ and $1 \%$ for $y>0.1$. This yields an uncertainty of $1 \%$ on the cross section measurements.

- A $y$-dependent error is assigned to the vertex finding efficiency: $15 \%$ for $y<0.06,7 \%$ for $0.06<y<0.1,4 \%$ for $0.1<y<0.2$ and $1 \%$ for $y>0.2$. This efficiency is estimated using pseudo-CC data yielding an uncertainty of $2.4 \%$ on the cross section measurements.

- An uncertainty of $0.5 \%$ accounts for the dependence of the acceptance correction on the PDFs used in the MC simulation.

- A $1.8 \%$ uncertainty on the trigger efficiency is determined based on the pseudo-CC data sample. The uncorrelated component of this uncertainty is $1 \%$. 
- An error of $0.8 \%$ is estimated for the QED radiative corrections. This accounts for missing contributions in the simulation of the lowest order QED effects and for the uncertainty on the higher order QED and EW corrections.

- In addition, there is a global uncertainty of $1.3 \%$ on the luminosity measurement for both the $R$ and $L$ data samples, of which $0.5 \%$ is considered as correlated.

The total systematic error is formed by adding the individual uncertainties in quadrature and amounts to about $4 \%$ on the cross section measurements.

The polarisation measurements have a relative uncertainty of $3.5 \%$ for the TPOL [25] and $1.6 \%$ for the LPOL [9] polarimeter and yield an absolute uncertainty on the mean polarisation of $\pm 0.7 \%$ for the $R$ sample and $\pm 1.1 \%$ for the $L$ sample. These are not included in the total systematic error on the cross section measurements, but are considered as independent uncertainties in a linear fit to the data.

\section{Results}

The measured integrated CC cross sections are quoted in the range $Q^{2}>400 \mathrm{GeV}^{2}$ and $y<0.9$ and are given in Table 1 and shown in Fig. 2. The measurement of the unpolarised total cross section in the same phase space based on $65.2 \mathrm{pb}^{-1}$ of data collected in 1999 and 2000 is also given. This measurement follows identically the procedure described in [5] but with the $Q^{2}$ cut adopted in this analysis. The systematic uncertainties of this unpolarised measurement are taken to be the same as in [5], with the exception of the QED radiative correction uncertainty, which has been reduced from $3 \%$ to $0.8 \%$. The measurements are compared to expectations of the Standard Model using the H1 PDF 2000 parametrisation. The uncertainty on the Standard Model expectations combines the uncertainties from experimental data used in the H1 PDF 2000 fit as well as model uncertainties [5].

\begin{tabular}{|r|c|c|}
\hline$P_{e}(\%)$ & $\sigma_{\mathrm{CC}}^{\text {tot }}(\mathrm{pb})$ & SM expectation $(\mathrm{pb})$ \\
\hline+33.6 & $35.6 \pm 1.5_{\text {stat }} \pm 1.4_{\text {sys }}$ & $35.1 \pm 0.6$ \\
0.0 & $28.4 \pm 0.8_{\text {stat }} \pm 0.8_{\text {sys }}$ & $26.3 \pm 0.4$ \\
-40.2 & $13.9 \pm 1.1_{\text {stat }} \pm 0.6_{\text {sys }}$ & $15.7 \pm 0.3$ \\
\hline
\end{tabular}

Table 1: Measured cross section values for $\sigma_{\mathrm{CC}}^{\text {tot }}\left(e^{+} p \rightarrow \bar{\nu} X\right)$ in the region $Q^{2}>400 \mathrm{GeV}^{2}$ and $y<0.9$ compared to the Standard Model (SM) expectation.

A linear fit to the polarisation dependence of the measured cross sections is performed taking into account the correlated systematic uncertainties between the measurements and is shown in Fig. 2. The fit provides a reasonable description of the data with a $\chi^{2}=4.4$ for one degree of freedom (dof). The result of the fit extrapolated to the point $P_{e}=-1$ yields a fully left handed charged current cross section of

$$
\sigma_{\mathrm{CC}}^{\mathrm{tot}}\left(P_{e}=-1\right)=-3.9 \pm 2.3_{\text {stat }} \pm 0.7_{\mathrm{sys}} \pm 0.8_{\mathrm{pol}} \mathrm{pb},
$$


where the quoted errors correspond to the statistical (stat), experimental (sys) and polarisationrelated (pol) systematic uncertainties. This extrapolated cross section is consistent with the Standard Model prediction of a vanishing cross section and corresponds to an upper limit on $\sigma_{\mathrm{CC}}^{\text {tot }}\left(P_{e}=-1\right)$ of $1.9 \mathrm{pb}$ at $95 \%$ confidence level (CL), as derived according to [26]. This result excludes the existence of charged currents involving right handed fermions mediated by a boson of mass below $208 \mathrm{GeV}$ at 95\% CL, assuming Standard Model couplings and a massless right handed $\nu_{e}$.

It is also possible to fit the measured cross sections by constraining the cross section at $P_{e}=-1$ to zero. This yields a cross section at $P_{e}=0$ of $27.5 \pm 0.6_{\text {stat }} \pm 0.9_{\text {sys }}$ pb with a $\chi^{2} /$ dof $=3.5$ and a negligible error due to the uncertainty on the polarisation measurement. The fitted value agrees well with the Standard Model expectation of $26.3 \pm 0.4 \mathrm{pb}$.

\section{Summary}

The first measurement has been performed of polarised $e^{+} p$ total charged current cross sections in the kinematic region of $Q^{2}>400 \mathrm{GeV}^{2}$ and $y<0.9$. The results presented here are based on data collected from collisions of unpolarised protons with unpolarised positrons and, for the first time, with longitudinal polarised positrons in left and right helicity states. The polarisation dependence of the charged current cross section has thus been established at HERA, extending previous tests of the chiral structure of the charged current interaction into the region of large, space-like $Q^{2}$. The data are found to be consistent with the absence of right handed charged currents as predicted by the Standard Model.

\section{Acknowledgements}

We are grateful to the HERA machine group whose outstanding efforts have made this experiment possible. We thank the engineers and technicians for their work in constructing and maintaining the $\mathrm{H} 1$ detector, our funding agencies for financial support, the DESY technical staff for continual assistance and the DESY directorate for support and for the hospitality which they extend to the non DESY members of the collaboration. 


\section{References}

[1] M. Klein and T. Riemann, Z. Phys. C 24 (1984) 151.

[2] A. Aktas et al. [H1 Collaboration], Submitted to Phys. Lett. B [hep-ex/0507080], DESY05-093.

[3] R. Beyer et al., Proceedings of the Workshop "Future Physics at HERA", vol. 1, eds. W. Buchmüller, G. Ingelman, A. De Roeck and R. Klanner, DESY (1996) 142;

R. Cashmore et al., ibid., 163.

[4] M. Jonker et al. [CHARM Collaboration], Phys. Lett. B 86 (1979) 229.

[5] C. Adloff et al. [H1 Collaboration], Eur. Phys. J. C 30 (2003) 1 [hep-ex/0304003].

[6] C. Adloff et al. [H1 Collaboration], Eur. Phys. J. C 21 (2001) 33 [hep-ex/0012053].

[7] A. Arbuzov, D.Y. Bardin, J. Bluemlein, L. Kalinovskaya and T. Riemann, Comput. Phys. Commun. 94, 128 (1996) [hep-ph/9511434].

[8] A.A. Sokolov and I.M. Ternov, Sov. Phys. Dokl. 8 No. 12 (1964) 1203.

[9] M. Beckmann et al., Nucl. Instrum. Meth. A 479 (2002) 334.

[10] D.P. Barber et al., Nucl. Instrum. Meth. A 329 (1993) 79.

[11] I. Abt et al. [H1 Collaboration], Nucl. Instrum. Meth. A 386 (1997) 310 and 348; R.D. Appuhn et al. [H1 SPACAL Group], Nucl. Instrum. Meth. A 386 (1997) 397.

[12] G.A. Schuler and H. Spiesberger, Proceedings of the Workshop "Physics at HERA", vol. 3, eds. W. Buchmüller, G. Ingelman, DESY (1992) 1419.

[13] G. Ingelman, Proceedings of the Workshop "Physics at HERA", vol. 3, eds. W. Buchmüller, G. Ingelman, DESY (1992) 1366.

[14] A. Kwiatkowski, H. Spiesberger and H.-J. Möhring, Comput. Phys. Commun. 69 (1992) 155.

[15] L. Lönnblad, Comput. Phys. Commun. 71 (1992) 15.

[16] T. Sjöstrand and M. Bengtsson, Comput. Phys. Commun. 43 (1987) 367.

[17] T. Sjöstrand, Comput. Phys. Commun. 82 (1994) 74.

[18] H.L. Lai et al. [CTEQ Collaboration], Eur. Phys. J. C 12 (2000) 375 [hep-ph/9903282].

[19] M. Glück, E. Reya and A. Vogt, Phys. Rev. D 46 (1992) 1973.

[20] R. Brun et al., GEANT3 User's Guide, CERN-DD/EE-84-1 (1987).

[21] B. Portheault, Ph.D. thesis (March 2005), LAL 05-05 (IN2P3/CNRS), Université de ParisSud XI, Orsay, available at http://www-h1.desy.de/publications/theses list.html. 
[22] A. Blondel and F. Jacquet, Proceedings of the Study of an ep Facility for Europe, ed. U. Amaldi, DESY (1979) 391.

[23] A. Aktas, Ph.D. thesis (August 2005), Hamburg University, available at http://wwwh1.desy.de/publications/theses_list.html.

[24] R. Plačakytè, Ph.D. thesis (in preparation), Max-Planck-Institut für Physik, Munich, will be available at http://www-h1.desy.de/publications/theses Jist.html.

[25] W. Lorenzon [HERMES Collaboration], DESY-HERMES-97-68, 7th International Workshop on Polarized Gas Targets and Polarized Beams, Urbana, IL, 18-22 Aug 1997; F. Corriveau et al. [TPOL Polarimeter Group], "A Calibration of the HERA Transverse Polarimeter for the 2003/2004 Data", available at http://www.desy.de/ pol2000/documents/documents.html.

[26] G.J. Feldman and R.D. Cousins, Phys. Rev. D 57 (1998) 3873 [physics/9711021]. 

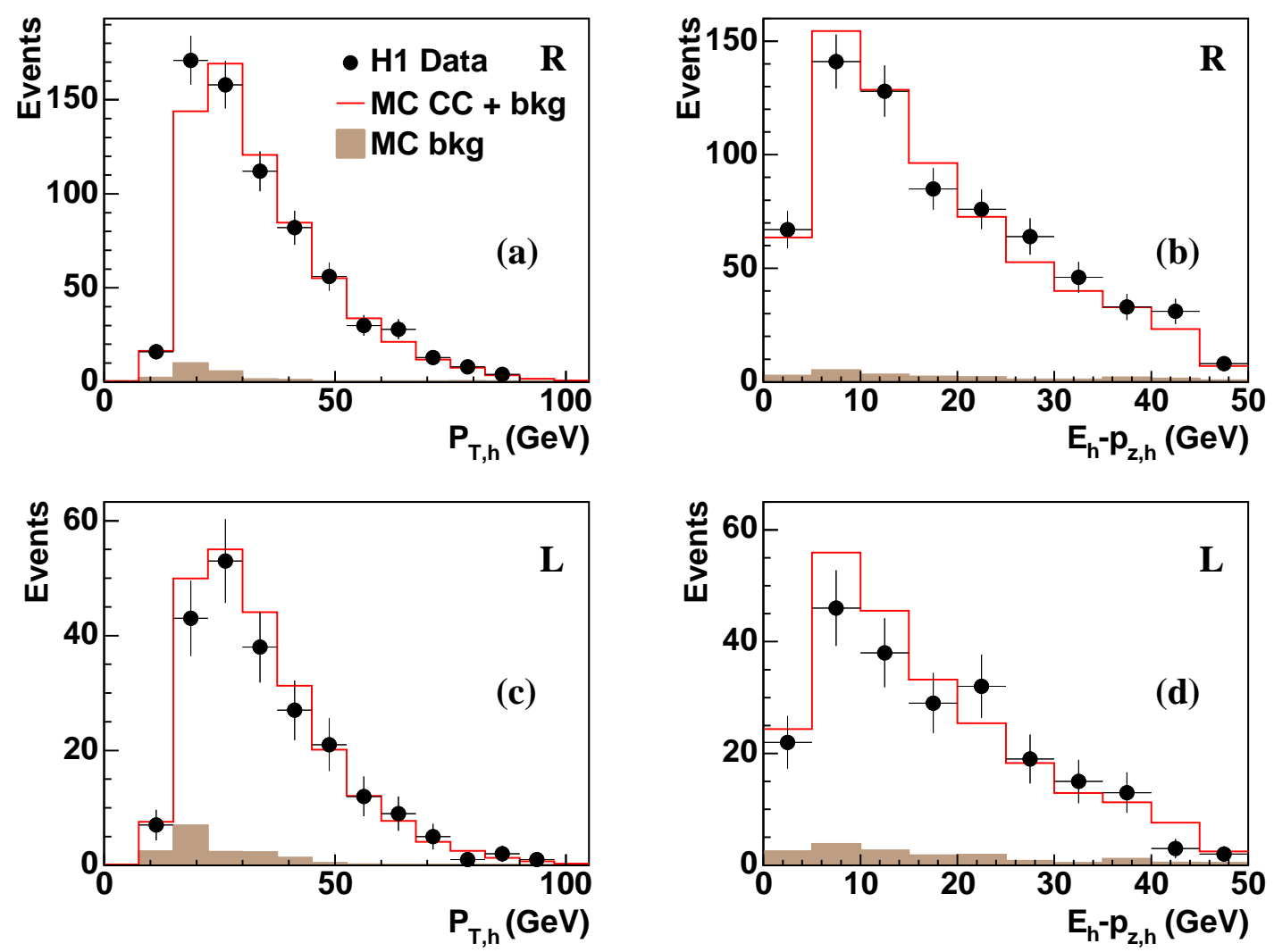

Figure 1: Distributions of $P_{T, h}(\mathrm{a}, \mathrm{c})$ and $E_{h}-p_{z, h}(\mathrm{~b}, \mathrm{~d})$ for the selected events in the right handed (R) and left handed (L) data sets. The Monte Carlo (MC) contributions from the charged current (CC) process and the ep background (bkg) processes are shown as open histograms with the latter contribution alone being shown as shaded histograms. 


\section{Charged Current $e^{+} p$ Scattering}

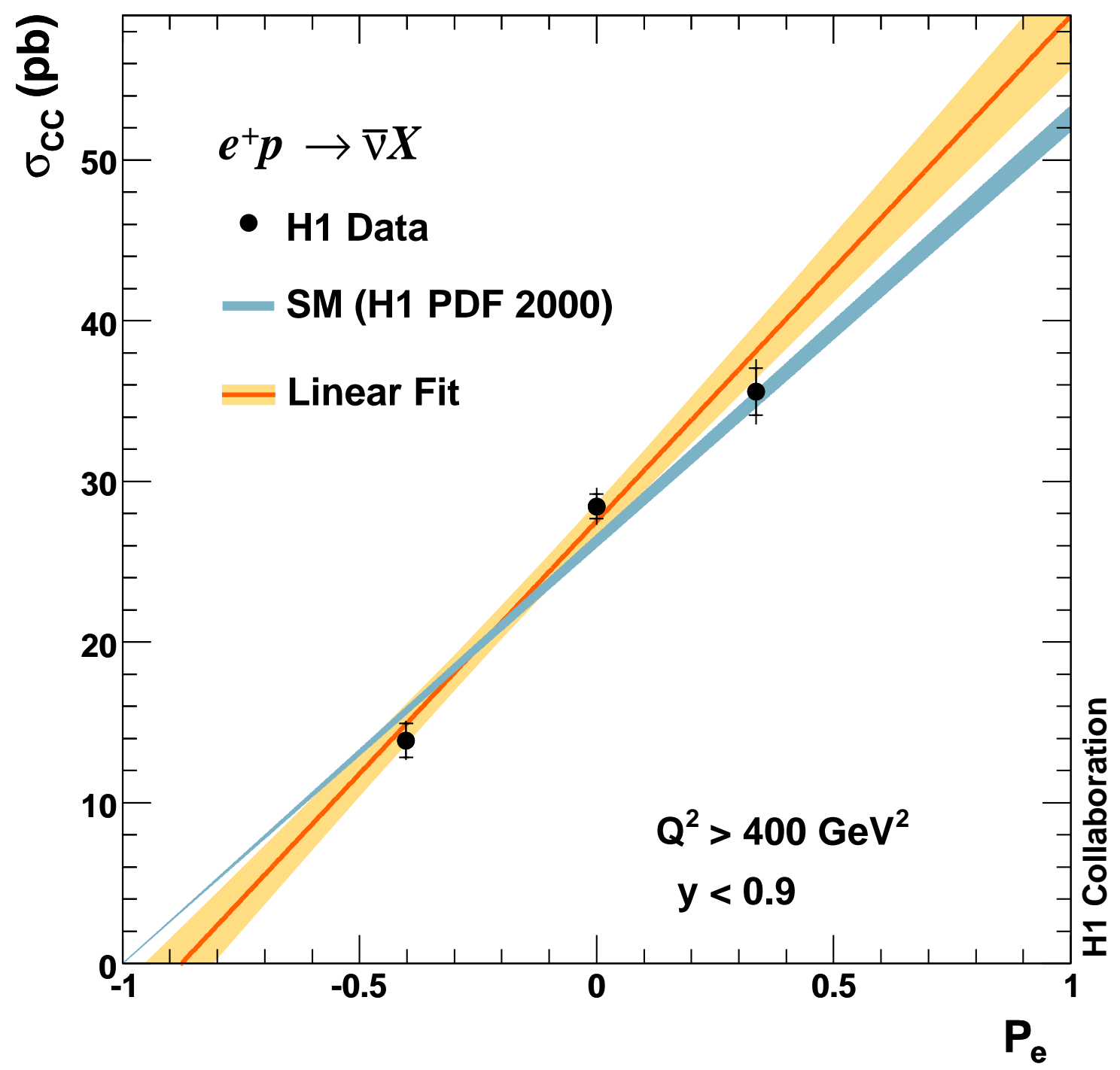

Figure 2: The dependence of the $e^{+} p \mathrm{CC}$ cross section on the lepton beam polarisation $P_{e}$. The inner and outer error bars represent respectively the statistical and total errors. The uncertainties on the polarisation measurement are smaller than the symbol size. The data are compared to the Standard Model prediction based on the H1 PDF 2000 parametrisation (dark shaded band). The light shaded band corresponds to the resulting one-sigma contour of a linear fit to the data shown as the central line. 